\title{
SPIRIT 2013
}

Evidence-Based Dentistry (2013) 14, 120. doi:10.1038/sj.ebd.6400973

We have often highlighted guidelines for the conduct and reporting of different study designs in this journal. One of the more recent additions to these guidelines is the SPIRIT 2013 statement (Standard Protocol Items: Recommendations for Interventional Trials). ${ }^{1}$

The SPIRIT statement provides a checklist of the recommended items for inclusion in a full clinical trial protocol. A paper that supports and explains the checklist and recommendations is freely available. ${ }^{1}$ The recommendation consists of a 33 item checklist. The checklist is split into a number of sections:

- Administrative information

- Introduction

- Methods - participants, interventions and outcomes

- Methods - assignment of interventions (for controlled trials)

- Methods - data collection management and analysis

- Methods - monitoring

- Ethics \& dissemination

- Appendices

These are explained in the Chan et al. paper. An online version of the checklist elements together with the explanations is also available on the SPIRIT Statement website www. spirit-statement.org) which has other resources (Fig. 1). With so many of the reviews we report on in this journal highlighting the need for better designed and better reported studies in all aspects of dentistry it is important that researchers should be aware of these internationally developed guidelines. In addition to this new guidance for protocols a large number of other guides have been available for a number of years that detail best reporting practice for a wide range of study designs. While a number of these guidance documents have their own website they can all be found on the Equator (Enhancing the QUAlity and Transparency Of health Research) Network website (www.equator-network.org). This is an excellent resource which has recently been updated, which has greatly improved the navigation of the site (Fig. 2).

1. Chan A-W, Tetzlaff JM, Gøtzsche PC, Altman DG Mann H, Berlin J, Dickersin K, Hróbjartsson A, Schulz KF, Parulekar WR, Krleža-Jerić K, Laupacis A, Moher D. SPIRIT 2013 Explanation and Elaboration: Guidance for protocols of clinical trials. Br Med J 2013; 346: e7586.

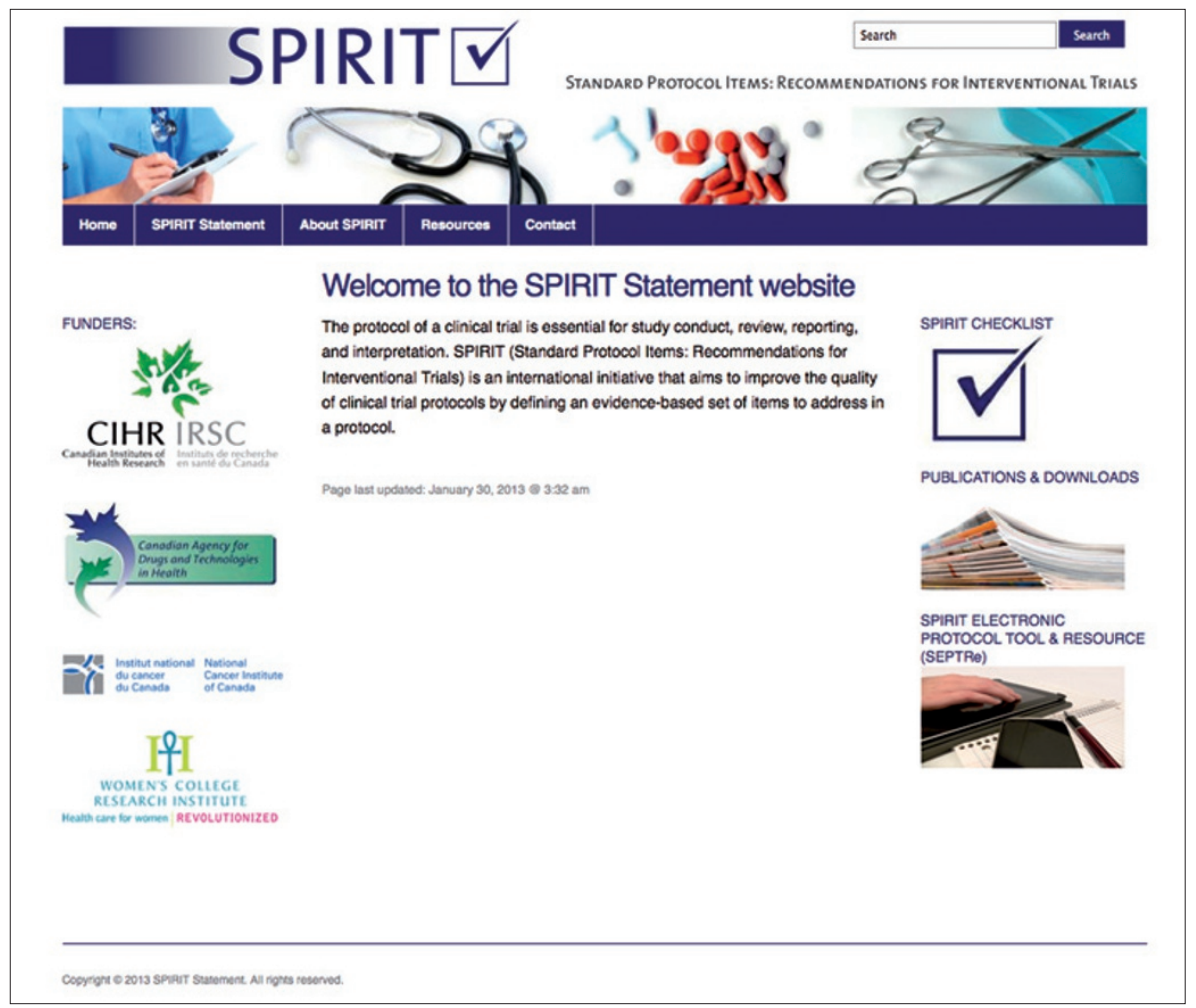

Figure 1. The SPIRIT Statement website

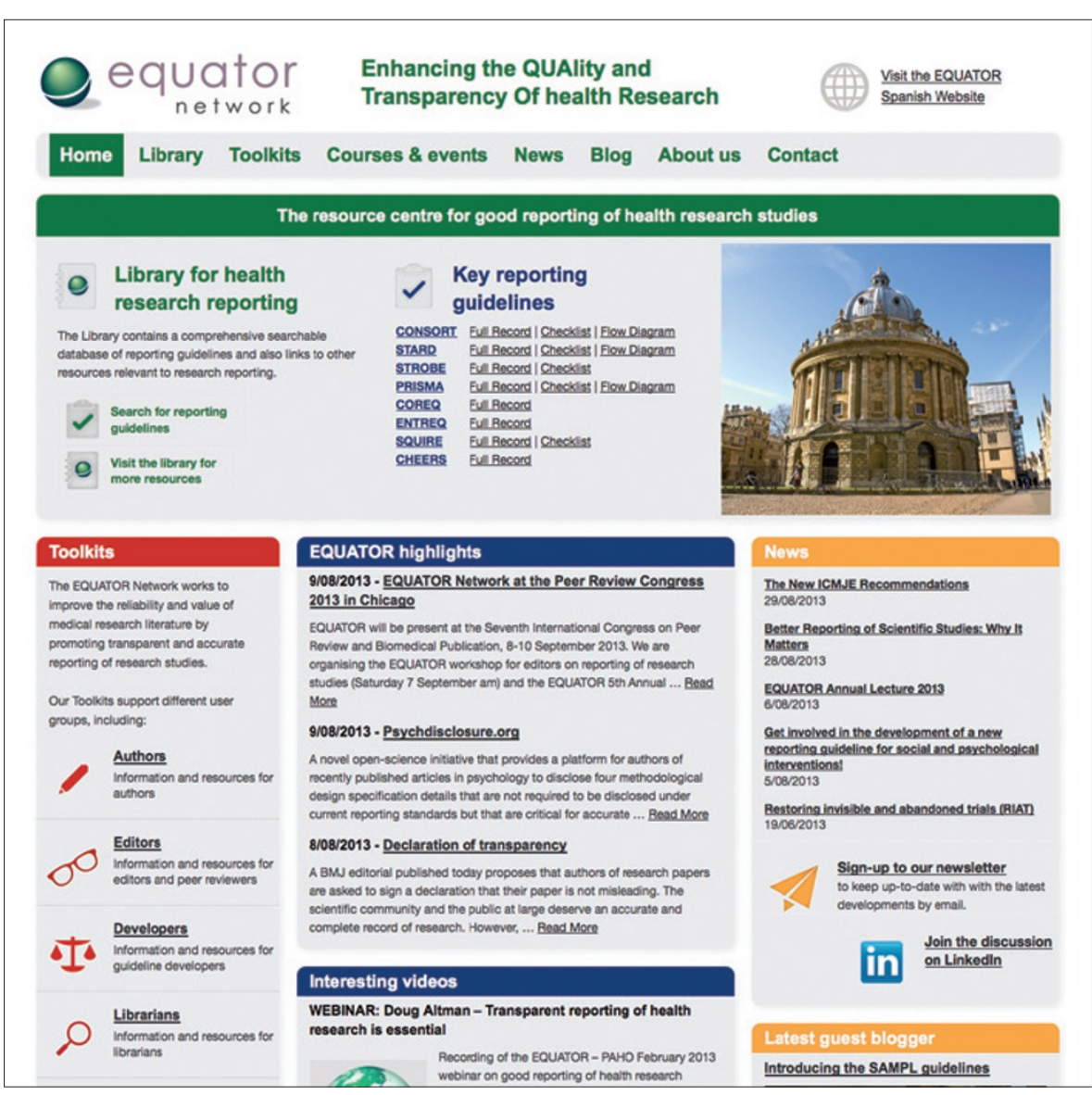

Figure 2. The equator network website home page 